\title{
Elbow Skin
}

National Cancer Institute

\section{Source}

National Cancer Institute. Elbow Skin. NCI Thesaurus. Code C52755.

The skin of the hinge joint that is located between the forearm and upper arm. 